

\title{
A new approach to Kostant's problem
}

\author{
Johan Kåhrström and Volodymyr Mazorchuk
}

\begin{abstract}
For every involution $\boldsymbol{w}$ of the symmetric group $S_{n}$ we establish, in terms of a special canonical quotient of the dominant Verma module associated with $\boldsymbol{w}$, an effective criterion to verify whether the universal enveloping algebra $U\left(\mathfrak{s l}_{n}\right)$ surjects onto the space of all ad-finite linear transformations of the simple highest weight module $L(\boldsymbol{w})$. An easy sufficient condition derived from this criterion admits a straightforward computational check (using a computer, for example). All this is applied to get some old and many new results, which answer the classical question of Kostant in special cases; in particular we give a complete answer for simple highest weight modules in the regular block of $\mathfrak{s l}_{n}, n \leq 5$.
\end{abstract}

\section{Introduction}

Let $\mathfrak{g}$ be a complex semisimple finite-dimensional Lie algebra with a fixed triangular decomposition,

$$
\mathfrak{g}=\mathfrak{n}_{-} \oplus \mathfrak{h} \oplus \mathfrak{n}_{+},
$$

and $U(\mathfrak{g})$ be its universal enveloping algebra. Then for every two $\mathfrak{g}$-modules $M$ and $N$ the space $\operatorname{Hom}_{\mathbb{C}}(M, N)$ may be viewed as a $U(\mathfrak{g})$-bimodule in the natural way, and, furthermore, also as a $\mathfrak{g}$-module under the adjoint action of $\mathfrak{g}$. The bimodule $\operatorname{Hom}_{\mathbb{C}}(M, N)$ has a sub-bimodule, usually denoted by $\mathscr{L}(M, N)$, which consists of all elements on which the adjoint action of $U(\mathfrak{g})$ is locally finite (see for example [Jantzen 1983, Kapitel 6]). Since $U(\mathfrak{g})$ itself consists of locally finite elements under the adjoint action, it naturally maps to $\mathscr{L}(M, M)$ for every $\mathfrak{g}$-module $M$, and the kernel of this map is obviously the annihilator $\operatorname{Ann}(M)$ of $M$ in $U(\mathfrak{g})$. The classical problem of Kostant (see for example [Joseph 1980]) is formulated in the following way:

For which $\mathfrak{g}$-modules $M$ is the natural injection $U(\mathfrak{g}) / \operatorname{Ann}(M) \hookrightarrow \mathscr{L}(M, M)$ surjective?

MSC2000: primary 17B10; secondary 17B35, 16E30.

Keywords: universal enveloping algebra, Kostant's problem, Kazhdan-Lusztig combinatorics. 
A (positive) answer to Kostant's problem is an important tool in the study of Goldie rank ratios [Joseph 1980] and in the study of induced modules, particularly generalized Verma modules, [Borho and Brylinski 1982; Miličić and Soergel 1997; Khomenko and Mazorchuk 2004; Mazorchuk and Stroppel 2008a]. ${ }^{1}$ A positive answer to Kostant's problem for certain highest weight modules allows one, for example, to give a unified irreducibility criterion for the structure of generalized Verma modules [Miličić and Soergel 1997; Khomenko and Mazorchuk 2004; MS 2008a]. A negative answer to Kostant's problem in the situation of [Borho and Brylinski 1982] results in the filtration of the appropriate quotient of the universal enveloping algebra not coinciding with that given by its embedding into differential operators.

Unfortunately, the complete answer to this problem is not even known for (simple) highest weight modules. The answer is known to be positive for Verma modules [Joseph 1980, Corollary 6.4] and for certain classes of simple highest weight modules [Gabber and Joseph 1981a, Theorem 4.4; Conze-Berline and Duflo 1977; McGovern 1994; Mazorchuk 2005, Theorem 1]. For simple highest weight modules in type A the answer is even known to be an invariant of a left cell; see [MS 2008a, Theorem 60]. However, already in [Joseph 1980, 9.5] it was shown that for some simple highest weight modules in type B the answer is negative (another example can be found in [Conze-Berline and Duflo 1977, 6.5]). Contrary to the popular belief that the answer is positive for simple highest weight modules in type A, it was recently shown in [MS 2008b, Theorem 13] that for the simple highest weight $\mathfrak{s l}_{4}$-module $L(r t)$, where $r$ and $t$ are two commuting simple reflections, the answer is negative.

The present paper is strongly inspired by this counterexample and is an attempt to analyze and generalize it. As for highest weight modules in type A the answer to Kostant's problem is an invariant of a left cell, and since every left cell of the symmetric group $S_{n}$ contains a unique involution [Sagan 2001, Chapter III], it is enough to solve Kostant's problem for all modules of the form $L(\boldsymbol{w})$, where $\boldsymbol{w} \in S_{n}$ is an involution. The counterexample in [MS 2008b, Theorem 13] was constructed relating the module $L(\boldsymbol{w})$ to a special quotient of the dominant Verma module, which in the following will be denoted by $D^{\hat{R}}$. This module is a canonical object of the category $\mathbb{O}_{0}^{\hat{\boldsymbol{R}}}$, which was used in [MS 2008a] to categorify Kazhdan-Lusztig cell modules. Furthermore, it is the unique quotient of the dominant Verma module, whose annihilator (in $U(\mathfrak{g})$ ) coincides with the annihilator of $L(\boldsymbol{w})$. The module $L(\boldsymbol{w})$ is the simple socle of $D^{\hat{R}}$ and thus both $L(\boldsymbol{w})$ and $D^{\hat{R}}$ are submodules of the indecomposable injective module $P^{\hat{R}}(\boldsymbol{w})$ in $\widehat{O}_{0}^{\hat{\boldsymbol{R}}}$, which also turns out to be projective.

\footnotetext{
${ }^{1}$ Henceforth we will abreviate "Mazorchuk and Stroppel" by MS.
} 
The main result of the present paper relates the solution of Kostant's problem for $L(\boldsymbol{w})$ to the structure of $D^{\hat{\boldsymbol{R}}}$ as follows:

Theorem 1. Kostant's problem has a positive answer for $L(w)$ if and only if every simple submodule of the cokernel of the canonical inclusion $D^{\hat{\boldsymbol{R}}} \subset P^{\hat{\boldsymbol{R}}}(\boldsymbol{w})$ has the form $L(x)$, where $x$ is some element from the right cell of $w$.

We will show that Theorem 1 can be used to answer Kostant's problem in many cases, in particular, to obtain many new results and reprove some old results. To prove this result we further develop the functorial approach to Kostant's problem from [Mazorchuk 2005; MS 2008a; 2008b]. The most interesting application of this theorem seems to be that it implies a sufficient condition for a negative answer to Kostant's problem, which is purely computational and can be realized as a relatively short and efficient program on a computer. Some further progress on Kostant's problem was recently made in [Mazorchuk 2009].

In Section 2 we collect all necessary preliminaries. The main results, including Theorem 1, are formulated in detail and proved in Section 3. In Section 4 we collect many applications, both theoretical and computational.

\section{Notation and preliminaries}

From now on we assume that $\mathfrak{g}=\mathfrak{s l}_{n}$ and the triangular decomposition is just the usual decomposition into the upper triangular, diagonal and lower triangular matrices. The symmetric group $S_{n}$ is the Weyl group $W$ for $\mathfrak{g}$ and hence $S_{n}$ acts on $\mathfrak{h}^{*}$ in the usual way $w \lambda$, and via the dot action $w \cdot \lambda=w(\lambda+\rho)-\rho$, where $\rho$ is half the sum of all positive (with respect to the above triangular decomposition) roots of the algebra $\mathfrak{g}$.

Let 0 denote the BGG category 0 [Bernstein et al. 1976] associated with the triangular decomposition above. For $w \in W$ we let $\Delta(w)$ denote the Verma module with highest weight $w \cdot 0, L(w)$ denote the simple head of $\Delta(w)$, and $P(w)$ denote the indecomposable projective cover of $L(w)$. The principal block $\mathrm{O}_{0}$ is the indecomposable direct summand of $\mathbb{0}$, which contains all $L(w), w \in S_{n}$.

For $w \in W$ we denote by $\theta_{w}$ the indecomposable projective functor on $\mathbb{O}_{0}$ associated with $w$. This functor is the unique (up to isomorphism) indecomposable direct summand of all possible functors that have the form $V \otimes_{\mathbb{C}-}: \mathbb{O} \rightarrow \mathbb{O}$, where $V$ is a finite-dimensional $\mathfrak{g}$-module that satisfies $\theta_{w} \Delta(e)=P(w)$, where $e$ is the identity element of $W$ [Bernstein and Gel'fand 1980, Section 3].

Denote by $\leq_{\mathrm{L}}$ and $\leq_{\mathrm{R}}$ the left and the right (pre)orders on $W$ respectively [Björner and Brenti 2005, Section 3]. For $x, y \in W$ we will write $x<_{\mathrm{L}} y$ provided that $x \leq_{\mathrm{L}} y$ and $y \leq_{\mathrm{L}} x$. We will use similar notation for $\leq_{\mathrm{R}}$. The left preorder coincides with the natural inclusion order on the set of annihilators of $L(w), w \in S_{n}$ [Jantzen 1983, 14.15]. The right one is obtained applying the involution $x \mapsto x^{-1}$. 
For a fixed right cell $\boldsymbol{R}$ set

$$
\hat{\boldsymbol{R}}=\left\{x \in W: x \leq_{\mathrm{R}} w \text { for some } w \in \boldsymbol{R}\right\}
$$

(this is simply the principal ideal (or cone) of $\left(S_{n}, \leq_{\mathrm{R}}\right)$, generated by the equivalence class $\boldsymbol{R})$, and denote by $\mathrm{O}_{0}^{\hat{\boldsymbol{R}}}$ the smallest full subcategory of $\mathrm{O}_{0}$ that contains all $L(w), w \in \hat{\boldsymbol{R}}$, and is closed under isomorphisms and extensions. The natural inclusion functor $\mathrm{O}_{0}^{\hat{R}} \rightarrow \mathrm{O}_{0}$ is obviously exact and hence has both a left adjoint $\mathrm{Z}_{0}^{\hat{\boldsymbol{R}}}: \mathrm{O}_{0} \rightarrow \mathrm{O}_{0}^{\hat{\boldsymbol{R}}}$ and a right adjoint $\hat{\mathrm{Z}}_{0}^{\hat{\boldsymbol{R}}}: \mathrm{O}_{0} \rightarrow \mathrm{O}_{0}^{\hat{\boldsymbol{R}}}[\mathrm{MS} 2008 \mathrm{a}, 5.1]$. The functor $\mathrm{Z}_{0}^{\hat{\boldsymbol{R}}}$ is just the functor of taking the maximal possible quotient that lies in $\mathrm{O}_{0}^{\hat{\boldsymbol{R}}}$; and the functor $\hat{Z}_{0}^{\hat{R}}$ is just the functor of taking the maximal possible submodule that lies in $\mathcal{O}_{0}^{\hat{\boldsymbol{R}}}$. All projective functors on $\mathrm{O}_{0}$ preserve $\mathcal{O}_{0}^{\hat{\boldsymbol{R}}}$, and both $\mathrm{Z}_{0}^{\hat{\boldsymbol{R}}}$ and $\hat{\mathrm{Z}}_{0}^{\hat{\boldsymbol{R}}}$ commute with $\theta_{w}$ for all $w \in W$ [MS 2008a, Lemma 19].

For $w \in \hat{\boldsymbol{R}}$ set $P^{\hat{\boldsymbol{R}}}(w)=\mathrm{Z}_{0}^{\hat{\boldsymbol{R}}} P(w)$ and $\Delta^{\hat{\boldsymbol{R}}}(w)=\mathrm{Z}_{0}^{\hat{\boldsymbol{R}}} \Delta(w)$. Then the modules $P^{\hat{\boldsymbol{R}}}(w), w \in \hat{\boldsymbol{R}}$, are exactly the indecomposable projective modules in $\mathcal{O}_{0}^{\hat{\boldsymbol{R}}}$. The module $P^{\hat{\boldsymbol{R}}}(w)$ is injective if and only if $w \in \boldsymbol{R}$ [MS 2008a, Section 5]. Let $\boldsymbol{w} \in \boldsymbol{R}$ be the unique involution in $\boldsymbol{R}$. Then $P^{\hat{\boldsymbol{R}}}(w)=\theta_{w} L(\boldsymbol{w})$ for any $w \in \boldsymbol{R}$; see [MS 2008b, Key statement]. By [MS 2008b, Lemma 8] we have the equality $\operatorname{dim} \operatorname{Hom}_{\mathfrak{g}}\left(P^{\hat{\boldsymbol{R}}}(e), P^{\hat{\boldsymbol{R}}}(\boldsymbol{w})\right)=1$. Denote by $D^{\hat{\boldsymbol{R}}}$ the image of the unique (up to a scalar) nonzero homomorphism from $P^{\hat{R}}(e)$ to $P^{\hat{R}}(\boldsymbol{w})$.

Conjecture 2. $D^{\hat{\boldsymbol{R}}}=P^{\hat{\boldsymbol{R}}}(e)$.

Define the following full subcategories in $\mathrm{O}_{0}^{\hat{\boldsymbol{R}}}$ :

$$
\begin{aligned}
& \mathscr{C}_{1}=\left\{M \in \mathcal{O}_{0}^{\hat{\boldsymbol{R}}}:[M: L(x)]>0 \text { implies } x<{ }_{\mathrm{R}} \boldsymbol{w}\right\}, \\
& \mathscr{C}_{2}=\left\{M \in \mathcal{O}_{0}^{\hat{\boldsymbol{R}}}: \operatorname{Hom}_{\mathfrak{g}}(L(x), M) \neq 0 \text { implies } x \in \boldsymbol{R}\right\}, \\
& \mathscr{C}_{3}=\left\{M \in \mathcal{O}_{0}^{\hat{\boldsymbol{R}}}: \operatorname{Hom}_{\mathfrak{g}}(M, L(x)) \neq 0 \text { implies } x \in \boldsymbol{R}\right\} .
\end{aligned}
$$

Let $M \in \mathscr{C}_{1}, N \in \mathscr{C}_{2}$ and $K \in \mathscr{C}_{3}$. Then none of the composition subquotients of $M$ occurs in the socle of $N$. Hence $\operatorname{Hom}_{\mathfrak{g}}(M, N)=0$. Similarly, none of the composition subquotients of $M$ occurs in the top of $K$. Hence $\operatorname{Hom}_{\mathfrak{g}}(K, M)=0$. The following result is based on a statement from [Joseph 1979].

Lemma 3. For all $w \in W$ and $i=1,2,3$, the functor $\theta_{w}$ preserves the category $\mathscr{b}_{i}$. Proof. Let $x<\mathrm{R} \boldsymbol{w}$ and $\boldsymbol{X}$ be the right cell of $x$. Then $L(x) \in \mathcal{O}_{0}^{\hat{X}}$ and hence $\theta_{w} L(x) \in \mathcal{O}_{0}^{\hat{\boldsymbol{X}}}$ (because, as mentioned above, $\theta_{w}$ preserves $\mathcal{O}_{0}^{\hat{\boldsymbol{X}}}$ ). Now for the category $\mathscr{C}_{1}$, the statement follows from the exactness of the functor $\theta_{w}$.

As noted before, in [MS 2008a, 5.1] it is shown that $P^{\hat{\boldsymbol{R}}}(w)$ is injective for any $w \in \boldsymbol{R}$. Since the socle of every $X \in \mathscr{C}_{2}$ consists, by definition, of $L(w), w \in \boldsymbol{R}$, it follows that the injective envelope of $X$ is projective. Since $\theta_{w}$ is both left and right adjoint to $\theta_{w^{-1}}$, it preserves the category of projective-injective modules in $0_{0}^{\hat{\boldsymbol{R}}}$. 
This and exactness of $\theta_{w}$ imply the statement for the category $\mathscr{C}_{2}$. For the category $\mathscr{C}_{3}$ the statement follows by duality.

Let $\mathscr{P}=\bigoplus_{w \in \boldsymbol{R}} P^{\hat{\boldsymbol{R}}}(w)$. For every $M \in \mathcal{O}_{0}^{\hat{\boldsymbol{R}}}$ let $I_{M}$ be some (minimal) injective envelope of $M$ and set

$$
M_{1}=\bigcap_{\substack{f \in \operatorname{Hom}_{\mathfrak{g}}\left(I_{M}, \mathscr{P}\right) \\ f(M)=0}} \operatorname{Ker}(f), \quad M_{1}^{\prime}=\bigcap_{f \in \operatorname{Hom}_{\mathfrak{g}}\left(M_{1}, \mathscr{P}\right)} \operatorname{Ker}(f),
$$

and $M_{2}=M_{1} / M_{1}^{\prime}$. Thus the module $M_{1}$ is the "maximal possible" nonsplit extension from a module from $\mathscr{C}_{1}$ to $M$, which does not affect the socle of $M$. The module $M_{1}^{\prime}$ is the maximal submodule of $M_{1}$ that belongs to $\mathscr{C}_{1}$. The correspondence $M \mapsto M_{2}$ is functorial and $M_{2}$ is called the partial approximation of $M$ with respect to the injective module $\mathscr{P}$ [Khomenko and Mazorchuk 2005, 2.5]. We denote by $\mathrm{A}: \mathrm{O}_{0}^{\hat{\boldsymbol{R}}} \rightarrow \mathrm{O}_{0}^{\hat{\boldsymbol{R}}}$ the corresponding functor of partial approximation. This functor is inspired by the realization of (Joseph's version of) Enright's functor obtained in [Khomenko and Mazorchuk 2005, Section 4]. However, one should mention that Enright's functor does not preserve $\mathrm{O}_{0}^{\hat{\boldsymbol{R}}}$ and hence is not suitable for our purposes. The functor $\mathrm{A}$ has the following properties:

Proposition 4. (i) A is left exact.

(ii) $\mathrm{A} M=0$ for any $M \in \mathscr{C}_{1}$.

(iii) A maps $\mathcal{O}_{0}^{\hat{\boldsymbol{R}}}$ to $\mathscr{C}_{2}$; in particular, A preserves the category $\mathscr{C}_{2}$.

(iv) The quotient map from $M$ to $M /\left(M \cap M_{1}^{\prime}\right)$ gives the natural transformation nat from the identity functor to $\mathrm{A}$.

(v) The kernel of nat coincides with the maximal submodule of $M$ that belongs to $\mathscr{C}_{1}$.

(vi) If $M \in \mathscr{C}_{2}$ and $I_{M}$ is the injective envelope of $M$, then $\mathrm{A} M$ is the maximal submodule of $I_{M}$ that contains $M$ and such that $\mathrm{A} M / M \in \mathscr{C}_{1}$.

(vii) If $M \in \mathscr{C}_{2}$, then $\mathrm{A} M \cong \mathrm{AA} M$.

Proof. Statement (i) is a part of [Khomenko and Mazorchuk 2005, Corollary 2]. If $M \in \mathscr{C}_{1}$, then $M_{1}^{\prime}=M$ and hence AM=0, proving (ii). As the module $M_{1}^{\prime}$ is the largest submodule of $M_{1}$ that belongs to $\mathscr{C}_{1}$, we get $M_{1} / M_{1}^{\prime} \in \mathscr{C}_{2}$, which proves (iii). For statement (iv) we refer to [Khomenko and Mazorchuk 2005, 2.5] and statement (v) follows from the definition of nat and the fact that $M_{1}^{\prime}$ is the largest submodule of $M_{1}$, which belongs to $\mathscr{C}_{1}$. If $M \in \mathscr{C}_{2}$, then $M_{1}^{\prime}=0$ and (vi) follows directly from the definition of A. Finally, (vii) follows from (vi). 


\section{The main results}

3.1. A criterion for testing Kostant's problem. According to Theorem 60 of [MS 2008a], the answer to Kostant's problem for $L(w), w \in W$, is an invariant of a left cell. Since every left cell has a unique involution, it is thus enough to study Kostant's problem for involutions in $W$. The main result of the paper is this:

Theorem 5. Let $\boldsymbol{w} \in W$ be an involution and $\boldsymbol{R}$ be the right cell of $W$, containing $\boldsymbol{w}$. Then the following conditions are equivalent:

(a) Kostant's problem has a positive solution for $L(w)$, that is, the inclusion

$$
U(\mathfrak{g}) / \operatorname{Ann}(M) \hookrightarrow \mathscr{L}(M, M)
$$

is surjective for $\mathfrak{g}=\mathfrak{s l}_{n}$ and $M=L(\boldsymbol{w})$.

(b) Every simple module occurring in the socle of the cokernel Coker of the natural inclusion $D^{\hat{\boldsymbol{R}} \hookrightarrow} P^{\hat{\boldsymbol{R}}}(\boldsymbol{w})$, has the form $L(x)$, where $x \in \boldsymbol{R}$ (that is, Coker belongs to $\mathscr{C}_{2}$ ).

The idea of the proof is to compare Kostant's problem for the modules $L(\boldsymbol{w})$ and $D^{\hat{\mathcal{R}}}$. The former is exactly the module for which we would like to solve Kostant's problem, while the latter is, by definition, a quotient of $\Delta(e)$, and hence Kostant's problem for it has a positive solution by [Jantzen 1983, 6.9(10)]. The relation between these two modules is again given by definition: $L(w)$ is the simple socle of $D^{\hat{\boldsymbol{R}}}$. So, to compare $\mathscr{L}(L(\boldsymbol{w}), L(\boldsymbol{w}))$ and $\mathscr{L}\left(D^{\hat{\boldsymbol{R}}}, D^{\hat{\boldsymbol{R}}}\right)$ one might first try to show that the modules $L(\boldsymbol{w})$ and $D^{\hat{R}}$ have the same annihilators, and then try to show that

$$
\operatorname{Hom}_{\mathfrak{g}}\left(L(\boldsymbol{w}), \theta_{w} L(\boldsymbol{w})\right)=\operatorname{Hom}_{\mathfrak{g}}\left(D^{\hat{\boldsymbol{R}}}, \theta_{w} D^{\hat{\boldsymbol{R}}}\right)
$$

for all $w \in W$. This would be enough to conclude that

$$
\mathscr{L}(L(\boldsymbol{w}), L(\boldsymbol{w}))=\mathscr{L}\left(D^{\hat{\boldsymbol{R}}}, D^{\hat{\boldsymbol{R}}}\right)
$$

by [Jantzen 1983, 6.8(3)], thus solving positively Kostant's problem for $L(\boldsymbol{w})$. The first step can be easily found in the literature.

Lemma 6. We have $\operatorname{Ann}_{U(\mathfrak{g})}(L(\boldsymbol{w}))=\operatorname{Ann}_{U(\mathfrak{g})}\left(D^{\hat{\boldsymbol{R}}}\right)$.

Proof. By [MS 2008b, Lemmata 6 and 8] and definitions, the module $L(\boldsymbol{w})$ is the simple socle of $D^{\hat{R}}$, and all other simple subquotients of $D^{\hat{R}}$ have the form $L(x)$ for some $x<_{\mathrm{R}} \boldsymbol{w}$; in particular, they all have Gel'fand-Kirillov dimension which is strictly smaller than that of $L(\boldsymbol{w})$ [Jantzen 1983, 10.11]. Hence $D^{\hat{\boldsymbol{R}}}$ is quasisimple in the sense of [Joseph 1980, 6.2], and thus the claim follows from [Joseph 1980, Proposition 6.2]. 
The best way to prove (1) would be to construct a functor that commutes with all $\theta_{w}$ and sends $L(\boldsymbol{w})$ to $D^{\hat{\boldsymbol{R}}}$. It turns out that the functor A defined above is the best possible candidate. We will now show that A commutes with $\theta_{w}$, and later on we will see that the answer to Kostant's problem is positive if and only if $\mathrm{A} L(\boldsymbol{w}) \cong D^{\hat{R}}$. So now let's do the work.

Lemma 7. For all $w \in W$ there is an isomorphism of functors

$$
\mathrm{A} \theta_{w} \cong \theta_{w} \mathrm{~A} \text {. }
$$

Proof. As A is left exact and $\theta_{w}$ is exact, both $\mathrm{A} \theta_{w}$ and $\theta_{w} \mathrm{~A}$ are left exact.

Let $I \in \mathbb{O}_{0}^{\hat{\boldsymbol{R}}}$ be injective. Consider the short exact sequence

$$
0 \rightarrow K \rightarrow I \stackrel{\text { nat }_{I}}{\longrightarrow} \mathrm{A} I \rightarrow 0,
$$

where $K$ is just the kernel of nat ${ }_{I}$. Since the socle of $\mathscr{P}$ coincides with $\bigoplus_{w \in \boldsymbol{R}} L(w)$, from the definition of A we have that $K \in \mathscr{C}_{1}$, while AI $\in \mathscr{C}_{2}$.

Applying $\theta_{w}$ to (2) and using Lemma 3 , we obtain that $\theta_{w} K \in \mathscr{C}_{1}$ and $\theta_{w} \mathrm{~A} I \in \mathscr{C}_{2}$. In particular, $\theta_{w} K$ is the maximal submodule of $\theta_{w} I$ that belongs to $\mathscr{C}_{1}$. Furthermore, the morphism $\theta_{w}\left(\right.$ nat $\left._{I}\right)$ is surjective.

At the same time, the module $\theta_{w} I$ is injective as $\theta_{w}$ is right adjoint to the exact functor $\theta_{w^{-1}}$. From the definition of A we have that the morphism nat $\theta_{\theta_{w} I}$ is surjective and that its kernel coincides with the maximal submodule of $\theta_{w} I$ that belongs to $\mathscr{C}_{1}$. In other words, the kernels of nat $\theta_{w} I$ and $\theta_{w}$ (nat $\left._{I}\right)$ coincide.

Now the statement of the lemma follows from [Khomenko and Mazorchuk 2005, Lemma 1], applied to the situation $\mathrm{F}=\mathrm{A} \theta_{w}, \mathrm{G}=\theta_{w} \mathrm{~A}$ and $\mathrm{H}=\theta_{w}$.

Set $\bar{D}^{\hat{R}}=\mathrm{A} L(\boldsymbol{w})$.

Lemma 8. (i) $\bar{D}^{\hat{\boldsymbol{R}}}$ is isomorphic to the maximal submodule of the module $P^{\hat{\boldsymbol{R}}}(\boldsymbol{w})$ that contains the socle of $P^{\hat{R}}(w)$ and such that all other composition subquotients of $\bar{D}^{\hat{R}}$ have the form $L(x)$, where $x<_{\mathrm{R}} \boldsymbol{w}$.

(ii) We have $D^{\hat{\boldsymbol{R}}} \subset \bar{D}^{\hat{\boldsymbol{R}}}$, and the condition (b) of Theorem 5 is equivalent to the equality $D^{\hat{R}}=\bar{D}^{\hat{R}}$.

Proof. As $P^{\hat{\boldsymbol{R}}}(\boldsymbol{w})$ is the injective envelope of $L(\boldsymbol{w})$, statement (i) follows from Proposition 4(vi). Claim (ii) follows from (i) and [MS 2008b, Lemmata 5 and 7].

To proceed we will need the following standard lemma:

Lemma 9. Let $X, Y \in \mathcal{O}$ be such that $\mathscr{L}(X, X) \subset \mathscr{L}(Y, Y)$ and

$$
\operatorname{dim} \operatorname{Hom}_{\mathfrak{g}}(X, \theta X)=\operatorname{dim} \operatorname{Hom}_{\mathfrak{g}}(Y, \theta Y)
$$

for any (indecomposable) projective functor $\theta$. Then $\mathscr{L}(X, X) \cong \mathscr{L}(Y, Y)$. 
Proof. Both $\mathscr{L}(X, X)$ and $\mathscr{L}(Y, Y)$ are Harish-Chandra bimodules for $\mathfrak{g}$ in the sense of [Jantzen 1983, Kapitel 6]. In particular, with respect to the adjoint action of $\mathfrak{g}$, these modules are direct sums of simple finite-dimensional $\mathfrak{g}$-modules, each occurring with a finite multiplicity [Jantzen 1983, Kapitel 6]. For every simple finite-dimensional module $V$ we compare the multiplicities of $V$ in $\mathscr{L}(X, X)$ and $\mathscr{L}(Y, Y)$ considered as $\mathfrak{g}$-modules with the adjoint action of $\mathfrak{g}$. By [Jantzen 1983, 6.8(3)] we have

$$
\left.\operatorname{Hom}_{\mathfrak{g}}(V, \mathscr{L}(X, X)) \cong \operatorname{Hom}_{\mathfrak{g}}(X \otimes V, X)\right) \cong \operatorname{Hom}_{\mathfrak{g}}\left(X, X \otimes V^{*}\right)
$$

First note that $\operatorname{Hom}_{\mathfrak{g}}\left(X, X \otimes V^{*}\right)$ is finite-dimensional for $X \in \mathbb{O}$. Since $V^{*} \otimes_{-}$is a projective functor and any projective functor is a unique direct sum of indecomposable projective functors, the claim follows from (3) and the assumptions.

We now state and show the key property of the functor A.

Lemma 10. For any $w \in W$ we have

$$
\operatorname{dim} \operatorname{Hom}_{\mathfrak{g}}\left(L(\boldsymbol{w}), \theta_{w} L(\boldsymbol{w})\right)=\operatorname{dim} \operatorname{Hom}_{\mathfrak{g}}\left(\bar{D}^{\hat{\boldsymbol{R}}}, \theta_{w} \bar{D}^{\hat{\boldsymbol{R}}}\right) .
$$

Proof. Since $L(\boldsymbol{w}) \in \mathscr{C}_{2}$, we have $\theta_{w} L(\boldsymbol{w}) \in \mathscr{C}_{2}$ by Lemma 3. Hence, by Proposition 4(v), we have that A does not annihilate $L(\boldsymbol{w})$, that A does not annihilate any simple submodule of $\theta_{w} L(\boldsymbol{w})$, and that A does not annihilate any homomorphism $\varphi: L(\boldsymbol{w}) \rightarrow \theta_{w} L(\boldsymbol{w})$. Therefore, applying A we obtain an inclusion

$$
\operatorname{Hom}_{\mathfrak{g}}\left(L(\boldsymbol{w}), \theta_{w} L(\boldsymbol{w})\right) \subset \operatorname{Hom}_{\mathfrak{g}}\left(\mathrm{A} L(\boldsymbol{w}), \mathrm{A} \theta_{w} L(\boldsymbol{w})\right)
$$

Using Lemma 7 and the definition of $\bar{D}^{\hat{\boldsymbol{R}}}$ we thus get the inclusion

$$
\operatorname{Hom}_{\mathfrak{g}}\left(L(\boldsymbol{w}), \theta_{w} L(\boldsymbol{w})\right) \subset \operatorname{Hom}_{\mathfrak{g}}\left(\bar{D}^{\hat{\boldsymbol{R}}}, \theta_{w} \bar{D}^{\hat{\boldsymbol{R}}}\right)
$$

On the other hand, consider the short exact sequence

$$
0 \rightarrow L(\boldsymbol{w}) \rightarrow \bar{D}^{\hat{\boldsymbol{R}}} \rightarrow C \rightarrow 0
$$

where $C$ is the cokernel. Applying the exact functor $\theta_{w}$ yields the short exact sequence

$$
0 \rightarrow \theta_{w} L(\boldsymbol{w}) \rightarrow \theta_{w} \bar{D}^{\hat{R}} \rightarrow \theta_{w} C \rightarrow 0 .
$$

Applying the bifunctor $\operatorname{Hom}_{\mathfrak{g}}\left({ }_{-},{ }_{-}\right)$from sequence (5) to sequence (6) yields the following commutative diagram with exact rows and columns: 


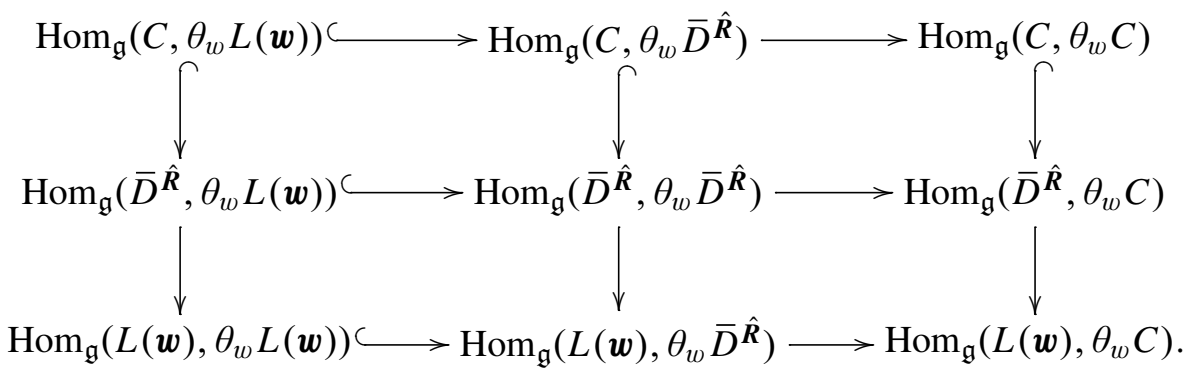

We have $C, \theta_{w} C \in \mathscr{C}_{1}$ by definitions and Lemmata 3 and 8 . We also have $L(\boldsymbol{w}) \in \mathscr{C}_{3}$. This yields $\operatorname{Hom}_{\mathfrak{g}}\left(L(\boldsymbol{w}), \theta_{w} C\right)=0$, which implies

$$
\operatorname{Hom}_{\mathfrak{g}}\left(L(\boldsymbol{w}), \theta_{w} L(\boldsymbol{w})\right)=\operatorname{Hom}_{\mathfrak{g}}\left(L(\boldsymbol{w}), \theta_{w} \bar{D}^{\hat{\boldsymbol{R}}}\right) .
$$

Since $C \in \mathscr{C}_{1}$ while $\bar{D}^{\hat{\boldsymbol{R}}}, \theta_{w} \bar{D}^{\hat{\boldsymbol{R}}} \in \mathscr{C}_{2}$ by definitions and Lemma 3 , we have $\operatorname{Hom}_{\mathfrak{g}}\left(C, \theta_{w} \bar{D}^{\hat{\boldsymbol{R}}}\right)=0$, which yields the inclusion

$$
\operatorname{Hom}_{\mathfrak{g}}\left(\bar{D}^{\hat{\boldsymbol{R}}}, \theta_{w} \bar{D}^{\hat{\boldsymbol{R}}}\right) \subset \operatorname{Hom}_{\mathfrak{g}}\left(L(\boldsymbol{w}), \theta_{w} \bar{D}^{\hat{\boldsymbol{R}}}\right) .
$$

This, together with the equality obtained in the previous paragraph, implies the opposite inclusion to (4); that is,

$$
\operatorname{Hom}_{\mathfrak{g}}\left(\bar{D}^{\hat{\boldsymbol{R}}}, \theta_{w} \bar{D}^{\hat{\boldsymbol{R}}}\right) \subset \operatorname{Hom}_{\mathfrak{g}}\left(L(\boldsymbol{w}), \theta_{w} L(\boldsymbol{w})\right) .
$$

The statement of the lemma follows.

Lemma 11. The inclusion $L(\boldsymbol{w}) \subset \bar{D}^{\hat{\boldsymbol{R}}}$ induces an isomorphism of $\mathfrak{g}$-bimodules as follows: $\mathscr{L}(L(\boldsymbol{w}), L(\boldsymbol{w})) \cong \mathscr{L}\left(\bar{D}^{\hat{\boldsymbol{R}}}, \bar{D}^{\hat{\boldsymbol{R}}}\right)$.

Proof. Applying the bifunctor $\mathscr{L}\left({ }_{-},{ }_{-}\right)$to (5), we get the following commutative diagram with exact rows and columns:

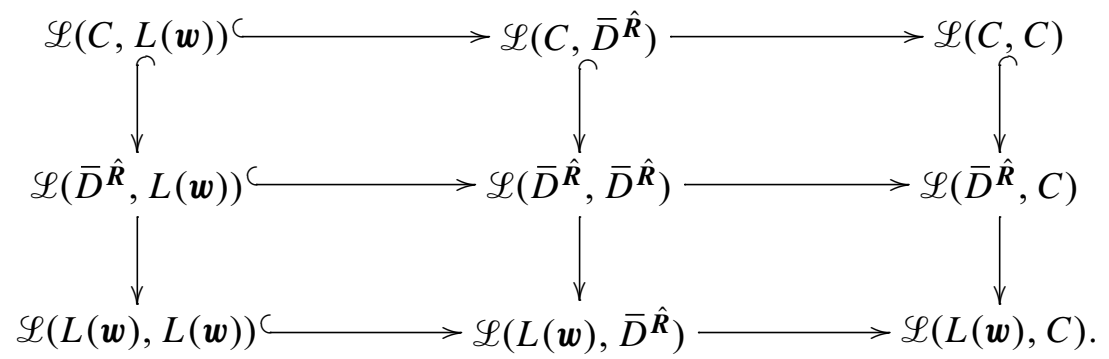

Note that for any $w \in W$ we have $C, \theta_{w} C \in \mathscr{C}_{1}$ by definitions and Lemma 3, while $L(\boldsymbol{w}) \in \mathscr{C}_{3}$. Hence from [Jantzen 1983, 6.8(3)], as in the proof of Lemma 9 we have $\mathscr{L}(L(\boldsymbol{w}), C)=0$ implying $\mathscr{L}(L(\boldsymbol{w}), L(\boldsymbol{w})) \cong \mathscr{L}\left(L(\boldsymbol{w}), \bar{D}^{\hat{\boldsymbol{R}}}\right)$. 
Since for any $w \in W$ we have $\bar{D}^{\hat{\boldsymbol{R}}}, \theta_{w} \bar{D}^{\hat{\boldsymbol{R}}} \in \mathscr{C}_{2}$ by the definitions and Lemma 3 , while $C \in \mathscr{C}_{1}$, from [Jantzen 1983, 6.8(3)] it follows that $\mathscr{L}\left(C, \bar{D}^{\hat{\boldsymbol{R}}}\right)=0$, implying $\mathscr{L}\left(\bar{D}^{\hat{\boldsymbol{R}}}, \bar{D}^{\hat{\boldsymbol{R}}}\right) \subset \mathscr{L}\left(L(\boldsymbol{w}), \bar{D}^{\hat{\boldsymbol{R}}}\right)$.

Hence $\mathscr{L}\left(\bar{D}^{\hat{\boldsymbol{R}}}, \bar{D}^{\hat{\boldsymbol{R}}}\right) \subset \mathscr{L}(L(\boldsymbol{w}), L(\boldsymbol{w}))$ and the proof is completed by applying Lemmata 9 and 10.

Proof of implication $(b) \Rightarrow(a)$ in Theorem 5. When (b) of Theorem 5 holds, then from Lemma 8(ii) we have $\bar{D}^{\hat{\boldsymbol{R}}}=D^{\hat{\boldsymbol{R}}}$. The module $D^{\hat{\boldsymbol{R}}}$ is a quotient of the dominant Verma module $\Delta(e)$, and hence $U(\mathfrak{g})$ surjects onto $\mathscr{L}\left(D^{\hat{\mathcal{R}}}, D^{\hat{\boldsymbol{R}}}\right)$ by [Jantzen 1983 , 6.9(10)]. Lemma 11 and diagram (7) now give the induced surjection of $U(\mathfrak{g})$ onto $\mathscr{L}(L(\boldsymbol{w}), L(\boldsymbol{w}))$. This completes the proof.

To prove the reverse implication, we will need some more properties of the functor A.

Lemma 12. (i) $\mathrm{A} \bar{D}^{\hat{\boldsymbol{R}}} \cong \bar{D}^{\hat{\boldsymbol{R}}}$.

(ii) $\mathrm{A} D^{\hat{\boldsymbol{R}}} \cong \bar{D}^{\hat{\boldsymbol{R}}}$.

(iii) For any $w \in W$ there is an isomorphism

$$
\operatorname{Hom}_{\mathfrak{g}}\left(D^{\hat{\boldsymbol{R}}}, \theta_{w} \bar{D}^{\hat{\boldsymbol{R}}}\right) \cong \operatorname{Hom}_{\mathfrak{g}}\left(\bar{D}^{\hat{\boldsymbol{R}}}, \theta_{w} \bar{D}^{\hat{\boldsymbol{R}}}\right) .
$$

Proof. We have $L(\boldsymbol{w}) \in \mathscr{C}_{2}$ and hence $\bar{D}^{\hat{\boldsymbol{R}}}=\mathrm{A} L(\boldsymbol{w}) \in \mathscr{C}_{2}$ by Proposition 4(iii). Therefore (i) follows from Proposition 4(vii).

Consider the short exact sequence

$$
0 \rightarrow D^{\hat{\boldsymbol{R}}} \rightarrow \bar{D}^{\hat{\boldsymbol{R}}} \rightarrow C \rightarrow 0
$$

where $C \in \mathscr{C}_{1}$ is the cokernel. We have $\mathrm{A} C=0$ by Proposition 4(ii). Now applying A to (8) and using (i) and the left exactness of A, we see that Proposition 4(i) yields statement (ii).

Since $C \in \mathscr{C}_{1}$ and $\bar{D}^{\hat{\boldsymbol{R}}}, \theta_{w} \bar{D}^{\hat{\boldsymbol{R}}} \in \mathscr{C}_{2}$, applying $\operatorname{Hom}_{\mathfrak{g}}\left({ }_{-}, \theta_{w} \bar{D}^{\hat{\boldsymbol{R}}}\right)$ to (8) yields the inclusion

$$
\operatorname{Hom}_{\mathfrak{g}}\left(\bar{D}^{\hat{\boldsymbol{R}}}, \theta_{w} \bar{D}^{\hat{\boldsymbol{R}}}\right) \subset \operatorname{Hom}_{\mathfrak{g}}\left(D^{\hat{\boldsymbol{R}}}, \theta_{w} \bar{D}^{\hat{\boldsymbol{R}}}\right) .
$$

On the other hand, both $D^{\hat{\boldsymbol{R}}}$ and $\theta_{w} \bar{D}^{\hat{\boldsymbol{R}}}$ belong to $\mathscr{C}_{2}$. Thus the functor A does not annihilate $D^{\hat{\boldsymbol{R}}}$, does not annihilate any submodule of $\theta_{w} \bar{D}^{\hat{\boldsymbol{R}}}$, and does not annihilate any morphism between these two modules (Proposition 4(v)). Hence, we have the inclusion

$$
\operatorname{Hom}_{\mathfrak{g}}\left(D^{\hat{\boldsymbol{R}}}, \theta_{w} \bar{D}^{\hat{\boldsymbol{R}}}\right) \subset \operatorname{Hom}_{\mathfrak{g}}\left(\mathrm{A} D^{\hat{\boldsymbol{R}}}, \mathrm{A} \theta_{w} \bar{D}^{\hat{\boldsymbol{R}}}\right) .
$$

Using (ii), Lemma 7 and (i) we obtain

$$
\operatorname{Hom}_{\mathfrak{g}}\left(\mathrm{A} D^{\hat{\boldsymbol{R}}}, \mathrm{A} \theta_{w} \bar{D}^{\hat{\boldsymbol{R}}}\right)=\operatorname{Hom}_{\mathfrak{g}}\left(\bar{D}^{\hat{\boldsymbol{R}}}, \theta_{w} \mathrm{~A} \bar{D}^{\hat{\boldsymbol{R}}}\right)=\operatorname{Hom}_{\mathfrak{g}}\left(\bar{D}^{\hat{\boldsymbol{R}}}, \theta_{w} \bar{D}^{\hat{\boldsymbol{R}}}\right),
$$


which implies that inclusion (9) is in fact an isomorphism. This completes the proof.

Proof of implication $(a) \Rightarrow(b)$ in Theorem 5. The inclusion $L(\boldsymbol{w}) \subset D^{\hat{\boldsymbol{R}}}$ induces the inclusion $\operatorname{Ann}_{U(\mathfrak{g})}\left(D^{\hat{\boldsymbol{R}}}\right) \subset \operatorname{Ann}_{U(\mathfrak{g})}(L(\boldsymbol{w}))$, which, in turn, induces the surjection

$$
U(\mathfrak{g}) / \operatorname{Ann}_{U(\mathfrak{g})}\left(D^{\hat{\boldsymbol{R}}}\right) \rightarrow U(\mathfrak{g}) / \operatorname{Ann}_{U(\mathfrak{g})}(L(\boldsymbol{w})) .
$$

Assume that (b) of Theorem 5 does not hold. As we have $\mathscr{L}\left(D^{\hat{\boldsymbol{R}}}, D^{\hat{\boldsymbol{R}}}\right) \cong$ $U(\mathfrak{g}) / \operatorname{Ann}_{U(\mathfrak{g})}\left(D^{\hat{\boldsymbol{R}}}\right)$ by [Jantzen 1983, 6.9(10)], from the latter formula and (10) it follows that the inequality

$$
\mathscr{L}\left(D^{\hat{\boldsymbol{R}}}, D^{\hat{\boldsymbol{R}}}\right) \subsetneq \mathscr{L}(L(\boldsymbol{w}), L(\boldsymbol{w})) \cong \mathscr{L}\left(\bar{D}^{\hat{\boldsymbol{R}}}, \bar{D}^{\hat{\boldsymbol{R}}}\right)
$$

would imply that the algebra $U(\mathfrak{g})$ does not surject onto $\mathscr{L}(L(\boldsymbol{w}), L(\boldsymbol{w})$ ) (since the image of $U(\mathfrak{g})$ coincides with $\left.\mathscr{L}\left(D^{\hat{\boldsymbol{R}}}, D^{\hat{\boldsymbol{R}}}\right)\right)$. Hence, what's left is to prove inequality (11).

We apply the bifunctor $\mathscr{L}\left({ }_{-},{ }_{-}\right)$to short exact sequence (8), where the cokernel $C \neq 0$ by Lemma 8(ii). Since $C \in \mathscr{C}_{1}$ and $D^{\hat{\boldsymbol{R}}}, \theta_{w} D^{\hat{\boldsymbol{R}}}, \bar{D}^{\hat{\boldsymbol{R}}}$ and $\theta_{w} \bar{D}^{\hat{\boldsymbol{R}}}$ are in $\mathscr{C}_{2}$ for all $w \in W$, by [Jantzen 1983, 6.8(3)] we obtain the following commutative diagram with exact rows and columns:

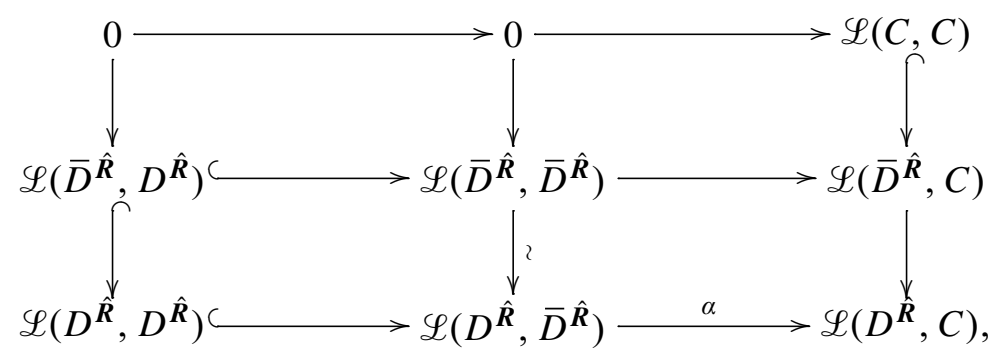

where the isomorphism in the second column follows from Lemma 12(iii). To complete the proof it is thus enough to show that the map $\alpha$ on diagram (12) is nonzero.

Pick some simple submodule $L(x) \subset C$ (recall once more that $C \neq 0$ by Lemma $8(\mathrm{ii})$ ). Using the adjointness and defining properties of projective functors, we have

$$
\begin{aligned}
\mathbb{C} & =\operatorname{Hom}_{\mathfrak{g}}\left(P^{\hat{\boldsymbol{R}}}(x), L(x)\right) \\
& =\operatorname{Hom}_{\mathfrak{g}}\left(\theta_{x} P^{\hat{\boldsymbol{R}}}(e), L(x)\right) \\
& =\operatorname{Hom}_{\mathfrak{g}}\left(P^{\hat{\boldsymbol{R}}}(e), \theta_{x^{-1}} L(x)\right) \\
& \subset \operatorname{Hom}_{\mathfrak{g}}\left(P^{\hat{\boldsymbol{R}}}(e), \theta_{x^{-1}} C\right) .
\end{aligned}
$$

Let $K$ be the kernel of the natural projection $P^{\hat{R}}(e) \rightarrow D^{\hat{R}}$ (note that $K \in \mathscr{C}_{1}$ by [MS 2008b, Lemmata 5 and 7]). Applying the bifunctor $\operatorname{Hom}_{\mathfrak{g}}\left({ }_{-},{ }_{-}\right)$from the 
short exact sequence

$$
0 \rightarrow K \rightarrow P^{\hat{\boldsymbol{R}}}(e) \rightarrow D^{\hat{\boldsymbol{R}}} \rightarrow 0
$$

to the short exact sequence

$$
0 \rightarrow \theta_{x^{-1}} D^{\hat{\boldsymbol{R}}} \rightarrow \theta_{x^{-1}} \bar{D}^{\hat{\boldsymbol{R}}} \rightarrow \theta_{x^{-1}} C \rightarrow 0,
$$

we obtain the following commutative diagram with exact rows and columns:

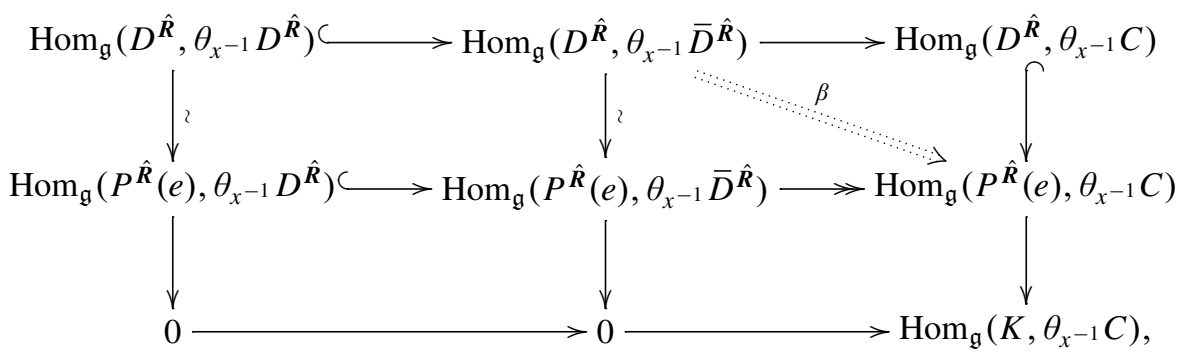

where the second row is exact as $P^{\hat{\boldsymbol{R}}}(e)$ is projective in $\mathrm{O}_{0}^{\hat{\boldsymbol{R}}}$, and the zeros in the third row follow from the fact that $K \in \mathscr{C}_{1}$ while $\theta_{x^{-1}} \bar{D}^{\hat{R}} \in \mathscr{C}_{2}$. From (13) it follows that the composition $\beta$ is a surjection onto a nonzero vector space, hence is a nonzero map. The map $\alpha$ contains, as a direct summand, the image of the map $\beta$ under the canonical isomorphism of [Jantzen 1983, 6.8]. Hence $\alpha \neq 0$. This completes the proof.

3.2. A sufficient condition for a negative answer. Let $\Lambda$ be the basic (that is, with one-dimensional simple modules) finite-dimensional associative algebra, whose module category is equivalent to $\mathscr{O}_{0}$. The algebra $\Lambda$ is Koszul [Soergel 1990], so we can fix the positive Koszul $\mathbb{Z}$-grading on $\Lambda$. Let $\Lambda$-gmod denote the category of finite-dimensional graded $\Lambda$-modules. For $w \in W$ let $e_{w}$ be the primitive idempotent of $\Lambda$, corresponding to $w$. Then we have the corresponding graded indecomposable projective module $\Lambda e_{w}$ (where the grading is induced from that on $\Lambda$ ). For $x \in \hat{\boldsymbol{R}}$, let $\mathrm{P}^{\hat{\boldsymbol{R}}}(x)$ denote the maximal quotient of $\Lambda e_{x}$ that belongs to $\hat{O}^{\hat{\boldsymbol{R}}}$ after forgetting the grading. The module $\mathrm{P}^{\hat{\boldsymbol{R}}}(x)$ is the standard graded lift of $P^{\hat{R}}(x)$ with head concentrated in degree zero (see [MS 2008a, 4.3]). Let L $(x)$ denote the simple quotient of $\mathrm{P}^{\hat{R}}(x)$. Then $\mathrm{L}(x)$ is the standard graded lift of the corresponding simple quotient (concentrated in degree zero). For $w \in W$ we denote by $\hat{\theta}_{w}$ the standard graded lift of the functors $\theta_{w}$ [Stroppel 2003, Section 8]. Finally, let $\boldsymbol{a}: W \rightarrow \mathbb{Z}$ denote Lusztig's $\boldsymbol{a}$-function [1985], which is uniquely determined by the properties that it is constant on the two-sided cells of $W$ and equals the length of the longest element $w_{0}^{\prime}$ (which belongs to this two-sided cell) of a parabolic subgroup of $W$. 
If $M$ is a graded module, then $M=\bigoplus_{i \in \mathbb{Z}} M_{i}$ is the decomposition of $M$ into a direct sum of graded components. As usual, for $k \in \mathbb{Z}$ we denote by $\langle k\rangle: \Lambda$-gmod $\rightarrow$ $\Lambda$-gmod the functor that shifts the grading such that $\mathrm{M}\langle k\rangle_{i}=\mathrm{M}_{i+k}$.

Lemma 13. Let $\boldsymbol{w} \in W$ be an involution and $\mathrm{M}=\hat{\theta}_{\boldsymbol{w}} \mathrm{L}(\boldsymbol{w})$. Then:

(i) $\mathrm{M}_{i}=0$ for all $i$ such that $|i|>\boldsymbol{a}(\boldsymbol{w})$.

(ii) $\mathrm{M}_{\boldsymbol{a}(\boldsymbol{w})}$ is the simple socle of $\mathrm{M}$ and is isomorphic to the module $\mathrm{L}(\boldsymbol{w})\langle-\boldsymbol{a}(\boldsymbol{w})\rangle$. Proof. Since $\boldsymbol{a}$ is an invariant of two-sided cells, by [MS 2008a, Theorem 18] we may without loss of generality assume that $\boldsymbol{w}$ is the maximal element of some parabolic subgroup. For such a $\boldsymbol{w}$, statement (i) follows immediately from [Stroppel 2003, Theorem 8.2]. Moreover, the same argument implies $M_{a(w)} \neq 0$.

As $\Lambda$ is positively graded and $M$ is injective (the latter follows from [MS 2008a, Section 5] and [MS 2008b, Key statement]), $M_{\boldsymbol{a}(\boldsymbol{w})} \neq 0$ must be the simple socle of M. On the other hand, we know that $\theta_{\boldsymbol{w}} L(\boldsymbol{w})=P^{\hat{\boldsymbol{R}}}(\boldsymbol{w})$. Hence the simple socle of $M$ is isomorphic (up to a shift of grading) to $L(w)$. Claim (ii) follows and the proof is complete.

Theorem 14. Let $w \in W$ be an involution and $\mathrm{M}=\hat{\theta}_{\boldsymbol{w}} \mathrm{L}(\boldsymbol{w})$. Assume that there exists $x \in W$ such that $x<_{\mathrm{R}} \boldsymbol{w}$ and

$$
[\mathrm{M}: \mathrm{L}(x)\langle 1-\boldsymbol{a}(\boldsymbol{w})\rangle]>\left[\mathrm{P}^{\hat{\boldsymbol{R}}}(e): \mathrm{L}(x)\langle 1-\boldsymbol{a}(\boldsymbol{w})\rangle\right] .
$$

Then Kostant's problem has a negative answer for $L(\boldsymbol{w})$.

Proof. Let $\mathrm{N}$ be the quotient of M modulo $D^{\hat{\boldsymbol{R}}}$. As $D^{\hat{\boldsymbol{R}}}$ is nonzero, it must contain the socle of M. Hence $\mathrm{N}_{i}=0$ for all $i \geq \boldsymbol{a}(\boldsymbol{w})$ by Lemma 13. By our assumption, $\mathrm{N}_{\boldsymbol{a}(\boldsymbol{w})-1}$ contains at least one copy of $\mathrm{L}(x)\langle 1-\boldsymbol{a}(\boldsymbol{w})\rangle$.

Since $\Lambda$ is positively graded and $N_{i}=0$ for all $i \geq \boldsymbol{a}(\boldsymbol{w})$, the space $\mathrm{N}_{\boldsymbol{a}(\boldsymbol{w})-1}$ belongs to the socle of $N$. Thus the condition (b) of Theorem 5 is not satisfied and the answer to Kostant's problem for $L(\boldsymbol{w})$ is negative by Theorem 5 .

Remark 15. As $\mathrm{P}^{\hat{\boldsymbol{R}}}(e)$ is a quotient of the graded dominant Verma module $\Delta(e)$, in Theorem 14 one could use a stronger assumption

$$
[\mathrm{M}: \mathrm{L}(x)\langle 1-\boldsymbol{a}(\boldsymbol{w})\rangle]>[\Delta(e): \mathrm{L}(x)\langle 1-\boldsymbol{a}(\boldsymbol{w})\rangle]
$$

with the same result.

Remark 16. The numerical condition of Theorem 14 is relatively easy to check (using a computer, for example), because it can be easily formulated in terms of Kazhdan-Lusztig combinatorics [Kazhdan and Lusztig 1979; Björner and Brenti 2005]. Via the standard categorification approach to 0 (see for example [MS 2008a, 3.4]), the characters of graded $\Lambda$-modules can be considered as elements of the Hecke algebra $\mathscr{H}$ of $W$ (such that Verma modules correspond to the standard basis 
of $\mathscr{H}$, projective modules correspond to the Kazhdan-Lusztig basis, and simple modules correspond to the dual Kazhdan-Lusztig basis). There are effective algorithms that allow one to multiply elements of $\mathscr{H}$ and to transform them from one of the mentioned basis to the other. Some of the applications presented in the next section are obtained using this approach.

Remark 17. The statement of Lemma 13 has a strong resemblance with [Mazorchuk 2007, Theorem 16], and is in some sense the Koszul dual of it (see the proof there for details).

\section{Applications}

In this section we present several applications of our main result, which show that it can be effectively applied in various situations. Unfortunately, we are still quite far from the complete answer.

\subsection{Kostant's problem for the socle of the dominant Verma module in a para-} bolic category. Let $\mathfrak{p} \subset \mathfrak{g}$ be a parabolic subalgebra containing $\mathfrak{h} \oplus \mathfrak{n}_{+}$, and let $\mathcal{O}_{0}^{\mathfrak{p}}$ be the corresponding parabolic subcategory of $\mathrm{O}_{0}$ in the sense of [Rocha-Caridi 1980]. Let $W^{\prime} \subset W$ be the Weyl group of the Levi factor of $\mathfrak{p}, w_{0}$ be the longest element in $W$ and $w_{0}^{\prime}$ be the longest element in $W^{\prime}$. Then

$$
\mathrm{O}_{0}^{\mathfrak{p}}=\mathrm{O}_{0}^{\hat{\boldsymbol{R}}},
$$

where $\boldsymbol{R}$ is the right cell of the element $w_{0}^{\prime} w_{0}$; see [MS 2008a, Remark 14]. Let $\boldsymbol{w}$ be the involution in $\boldsymbol{R}$. The following result is mentioned at the end of [McGovern 1994, Section 3] without proof.

Corollary 18. Kostant's problem has a positive answer for $L(\boldsymbol{w})$.

Proof. The category $\mathcal{O}_{0}^{\mathfrak{p}}$ is known to be a highest weight category in the sense of [Cline et al. 1988]. Thus any projective-injective module in $\mathcal{O}_{0}^{\mathfrak{p}}$ is tilting in the sense of [Ringel 1991]; in particular, it has a filtration by standard modules (that is, generalized Verma modules, induced from simple finite-dimensional $\mathfrak{p}$-modules). In particular, the dominant standard module $P^{\hat{R}}(e)$ is a submodule of $P^{\hat{R}}(w)$, and the cokernel of this inclusion again has a filtration by standard modules. Since all standard modules belong to $\mathscr{C}_{2}$ by [Irving 1985] (see also [MS 2008c, Theorem 5.1] for a short argument), we obtain that the condition (b) of Theorem 5 is satisfied and hence Kostant's problem has a positive answer for $L(\boldsymbol{w})$ by Theorem 5 .

Remark 19. The inclusion $P^{\hat{\boldsymbol{R}}}(e) \subset P^{\hat{\boldsymbol{R}}}(\boldsymbol{w})$ implies that Conjecture 2 is true if $\boldsymbol{R}$ contains some $w_{0}^{\prime} w_{0}$.

Remark 20. Corollary 18 holds for all semisimple finite-dimensional Lie algebras. 


\subsection{Kostant's problem for $L(s)$, where $s$ is a simple reflection.}

Corollary 21 [Mazorchuk 2005]. Let $s \in W$ be a simple reflection. Then Kostant's problem has a positive answer for $L(s)$.

Proof. The only element of $W$ that is strictly smaller than $s$ with respect to the order $<_{\mathrm{R}}$ is the identity element $e$, as, by adjointness,

$$
\operatorname{dim} \operatorname{Hom}_{\mathfrak{g}}\left(P^{\hat{\boldsymbol{R}}}(e), \theta_{s} L(s)\right)=\operatorname{dim} \operatorname{Hom}_{\mathfrak{g}}\left(P^{\hat{\boldsymbol{R}}}(s), L(s)\right)=1,
$$

the module $L(e)$ occurs in $\theta_{s} L(s)$ with multiplicity one, and hence $L(e)$ does not occur in the cokernel of the inclusion $D^{\hat{\boldsymbol{R}}} \subset \theta_{s} L(s)$ at all. Therefore the condition (b) of Theorem 5 is obviously satisfied and hence Kostant's problem has a positive answer for $L(s)$ by Theorem 5 .

\subsection{Kostant's problem for $L(s t)$, where $s$ and $t$ are commuting simple reflec-} tions. Here we generalize the counterexample, constructed in [MS 2008b, Section 5]. Let $s_{i}=(i, i+1), i=1, \ldots, n-1$, be the $i$ th simple reflection in $W$. We recall that for a simple reflection $s \in W$ and any $x \in W$ such that $x s<x$ with respect to the Bruhat order, we have that the module $\hat{\theta}_{s} \mathrm{~L}(x)$ is self-dual with simple head and socle, and we moreover have the following graded picture of this module (the middle row is in degree 0 , and the arrows schematically represent the action of elements from the algebra $\Lambda$ ):

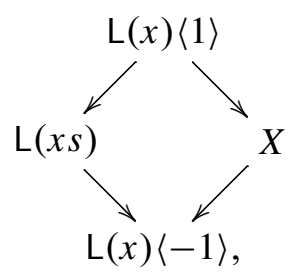

where $X$ is a direct sum of modules $L(y)$ such that $y s>y$, and the multiplicity of $L(y)$ in $X$ is $\mu(x, y)$, where $\mu$ is Kazhdan and Lusztig's $\mu$-function [1979]. The latter is a standard corollary of the now proved Kazhdan-Lusztig conjecture in the equivalent form given by Vogan (see [Kazhdan and Lusztig 1979; Gabber and Joseph 1981b; Vogan 1979]). We also refer to Remark 16 and to [Stroppel 2003, Section 8] for the appropriate graded reformulation.

Corollary 22. Let $s_{i}$ and $s_{j}$ be two commuting different simple reflections in $W$ (that is, $|i-j|>1)$. Then Kostant's problem has a positive answer for $L\left(s_{i} s_{j}\right)$ if and only if $|i-j|>2$.

Proof. Without loss of generality we assume $j>i$. Let $\boldsymbol{R}_{e}=\{e\}, \boldsymbol{R}_{i}$ denote the right cell of $s_{i}, \boldsymbol{R}_{j}$ denote the right cell of $s_{j}$, and $\boldsymbol{R}$ denote the right cell of 
$s_{i} s_{j}$. Then the Hasse diagram of $<_{\mathrm{R}}$ on the set $\left\{\boldsymbol{R}_{\boldsymbol{e}}, \boldsymbol{R}_{i}, \boldsymbol{R}_{j}, \boldsymbol{R}\right\}$, where $\boldsymbol{R}$ is the maximum element, is as follows:

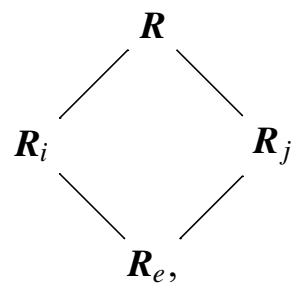

and we further have

$$
\begin{aligned}
\boldsymbol{R}_{i} & =\left\{s_{i}, s_{i} s_{i-1}, \ldots, s_{i} s_{i-1} \ldots s_{1}, s_{i} s_{i+1}, \ldots, s_{i} s_{i+1} \ldots s_{n-1}\right\} \\
\boldsymbol{R}_{j} & =\left\{s_{j}, s_{j} s_{j-1}, \ldots, s_{j} s_{j-1} \ldots s_{1}, s_{j} s_{j+1}, \ldots, s_{j} s_{j+1} \ldots s_{n-1}\right\} .
\end{aligned}
$$

A direct calculation gives $\theta_{s_{i}} \theta_{s_{j}}=\theta_{s_{i} s_{j}}=\theta_{s_{j}} \theta_{s_{i}}$.

Assume first that $j=i+2$. Since both $s_{i} s_{i+2}$ and $s_{i} s_{i+1} s_{i+2}$ are Boolean elements of $W$ (in the sense of [Marietti 2006]), we have that the Kazhdan-Lusztig polynomial $P_{s_{i} s_{i+2}, s_{i} s_{i+1} s_{i+2}}(q)=1$ by [Marietti 2006, Theorem 5.4] and hence $\mu\left(s_{i} s_{i+2}, s_{i} s_{i+1} s_{i+2}\right)=1$ as well by definition. This yields

$$
\operatorname{Ext}_{\overparen{O}}^{1}\left(L\left(s_{i} s_{i+2}\right), L\left(s_{i} s_{i+1} s_{i+2}\right)\right) \neq 0,
$$

and thus $L\left(s_{i} s_{i+1} s_{i+2}\right)$ occurs as a composition subquotient in $\theta_{s_{i}} L\left(s_{i} s_{i+2}\right)$ (as a direct summand of $X$ in (14)). Applying (14), we get that $\mathrm{L}\left(s_{i} s_{i+1} s_{i+2}\right)\langle-1\rangle$ occurs as a composition subquotient in $\hat{\theta}_{s_{i} s_{i+2}} \mathrm{~L}\left(s_{i} s_{i+2}\right)$. Note that we have

$$
s_{i} s_{i+1} s_{i+2}<_{\mathrm{R}} s_{i} s_{i+2} .
$$

At the same time, from [Dixmier 1996, Lemma 7.2.5], it follows that $\mathrm{P}^{\hat{R}}(e)_{1}$ contains only composition subquotients of the form $\mathrm{L}\left(s_{k}\right)\langle-1\rangle, k=1, \ldots, n-1$. Hence the numerical assumption of Theorem 14 is satisfied and therefore the answer to Kostant's problem for $L\left(s_{i} s_{i+2}\right)$ is negative by Theorem 14 .

If $j>i+2$, a similar application of [Marietti 2006, Theorem 5.4] yields

$$
\mu\left(s_{i} s_{j}, s_{i} s_{i+1} \ldots s_{j-1} s_{j}\right)=0 \quad \text { and } \quad \mu\left(s_{i} s_{j}, s_{j} s_{j-1} \ldots s_{i+1} s_{i}\right)=0 .
$$

The only other elements of $\boldsymbol{R}_{i}$ and $\boldsymbol{R}_{j}$, comparable with $s_{i} s_{j}$ with respect to the Bruhat order, are $s_{i}$ and $s_{j}$ respectively. Because of (14), this means that the module $\hat{\theta}_{s_{i}} \mathrm{~L}\left(s_{i} s_{j}\right)$ looks as follows:

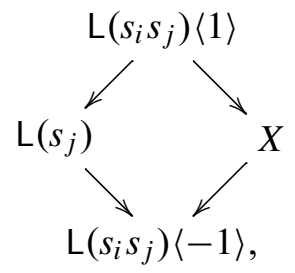


where $X$ is a direct sum of simple modules $L(y), y \in \boldsymbol{R}$. Applying now $\hat{\theta}_{s_{j}}$ and using (14) again we obtain the following graded filtration for the module $\hat{\theta}_{s_{i} s_{j}} \mathrm{~L}\left(s_{i} s_{j}\right)$ :

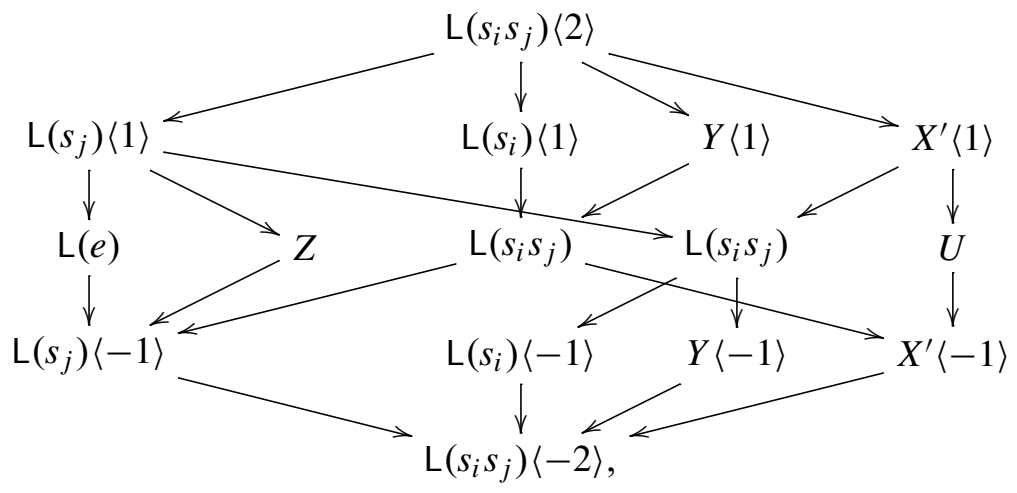

where $Z$ is a direct sum of simple modules of the form $L(y), y \in \boldsymbol{R}_{j} ; Y$ is a direct sum of simples modules of the form $L(y), y \in \boldsymbol{R}$; and $X^{\prime}$ is a direct summand of $X$. Note that the arrows on (15) (which are supposed to schematically represent the action of $\Lambda$ ) show only the part of the action, which obviously comes from (14), but they do not show the whole action. From [Dixmier 1996, Lemma 7.2.5] it follows that the module $\mathrm{D}^{\hat{R}}$ looks as follows:

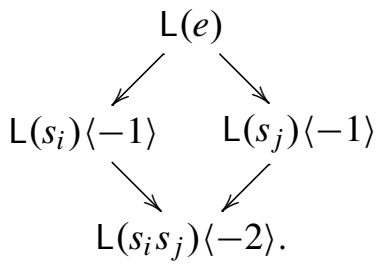

Now we have to analyze (15) to determine the cokernel $C$ of the inclusion $\mathrm{D}^{\hat{\boldsymbol{R}}} \subset$ $\hat{\theta}_{s_{i} s_{j}} \mathrm{~L}\left(s_{i} s_{j}\right)$. C obviously contains both $Y\langle-1\rangle$ and $X^{\prime}\langle-1\rangle$, but all direct summands of these modules have the form $L(y), y \in \boldsymbol{R}$, by the above. None of the simple subquotients of $U$ can occur as a submodule in $C$ by (14). Similarly one excludes $\mathrm{L}\left(s_{i}\right)\langle 1\rangle$ and $\mathrm{L}\left(s_{i}\right)\langle 1\rangle$. All simple submodules in $Z$ have the form $L(y)$, $y \in \boldsymbol{R}_{j}$. Considering $\hat{\theta}_{s_{i} s_{j}} \mathrm{~L}\left(s_{i} s_{j}\right)=\hat{\theta}_{s_{i}} \hat{\theta}_{s_{j}} \mathrm{~L}\left(s_{i} s_{j}\right)$ and using the same arguments as above, one shows that none of the simple submodules of $Z$ belongs to $C$. Hence $C$ contains only simple modules of the form $L(y), y \in \boldsymbol{R}$. Thus the condition (b) of Theorem 5 is satisfied and therefore Kostant's problem has a positive answer for $L\left(s_{i} s_{j}\right)$ by Theorem 5 . This completes the proof.

\subsection{Kostant's problem for $\mathfrak{s l}_{n}, n \leq 3$.}

Proposition 23. Assume that $n \leq 3$ and $w \in W$. Then Kostant's problem has a positive answer for $L(w)$. 
Proof. The statement is trivial for $n=1$. In the case $n=2$ for $w=e$ the statement follows from [Jantzen 1983, 6.9(10)] (as $L(e)$ is a quotient of the dominant Verma module) and for $w=s_{1}$ it follows from [Joseph 1980, Corollary 6.4] (as $L\left(s_{1}\right)$ is a Verma module).

Finally, in the case $n=3$ for $w=e$ the statement follows, as above, from [Jantzen 1983, 6.9(10)], for $w=s_{1}, s_{2}$ it follows from Corollary 21, for $w=s_{1} s_{1}, s_{2} s_{1}$ it follows from [Gabber and Joseph 1981a, Theorem 4.4], and, finally, for $w=s_{1} s_{2} s_{1}$ it follows, as above, from [Joseph 1980, Corollary 6.4].

\subsection{Kostant's problem for $\mathfrak{s l}_{4}$.}

Proposition 24. Assume that $n=4$ and $w \in W$. Then Kostant's problem has a positive answer for $L(w)$ if and only if $w \neq s_{1} s_{3}, s_{2} s_{1} s_{3}$.

Proof. The group $S_{4}$ has 10 involutions: $e, s_{1}, s_{2}, s_{3}, s_{1} s_{3}, s_{1} s_{2} s_{1}, s_{3} s_{2} s_{3}, s_{2} s_{1} s_{3} s_{2}$, $s_{1} s_{2} s_{3} s_{2} s_{1}$, and $s_{2} s_{1} s_{2} s_{3} s_{2} s_{1}$. The module $L(e)$ is a quotient of the dominant Verma module, and hence for $L(e)$ the claim follows from [Jantzen 1983, 6.9(10)]. The module $L\left(s_{2} s_{1} s_{2} s_{3} s_{2} s_{1}\right)$ is a Verma module and hence for this module the claim follows from [Joseph 1980, Corollary 6.4]. For $L\left(s_{1}\right), L\left(s_{2}\right), L\left(s_{3}\right)$ the claim follows from Corollary 21. The left cell of each of the elements $s_{1} s_{2} s_{1}, s_{3} s_{2} s_{3}$, $s_{2} s_{1} s_{3} s_{2}, s_{1} s_{2} s_{3} s_{2} s_{1}$ contains an element of the form $w_{0}^{\prime} w_{0}$, where $w_{0}^{\prime}$ is the longest element of some parabolic subgroup. Hence for $L\left(s_{1} s_{2} s_{1}\right), L\left(s_{3} s_{2} s_{3}\right), L\left(s_{2} s_{1} s_{3} s_{2}\right)$ and $L\left(s_{1} s_{2} s_{3} s_{2} s_{1}\right)$ the claim follows from [Gabber and Joseph 1981a, Theorem 4.4] and [MS 2008a, Theorem 60]. Finally, for $L\left(s_{1} s_{3}\right)$ the claim follows from Corollary 22 (or [MS 2008b, Theorem 13]). Note that the answer is negative only in the case of $L\left(s_{1} s_{3}\right)$. The left cell of $s_{1} s_{3}$ contains one more element, namely $s_{2} s_{1} s_{3}$. The statement of the proposition now follows from [MS 2008a, Theorem 60].

\subsection{Kostant's problem for $\mathfrak{s l}_{5}$.}

Proposition 25. Assume that $n=5$ and $w \in W$. Then Kostant's problem has a positive answer for $L(w)$ if and only if $w$ does not belong to the left cells containing one of the following involutions: $s_{1} s_{3}, s_{2} s_{4}, s_{2} s_{3} s_{2}, s_{1} s_{2} s_{1} s_{4}$ or $s_{1} s_{3} s_{4} s_{3}$.

Proof. The group $S_{5}$ has 26 involutions. As above, Kostant's problem has a positive answer for $L(e)$ since it is a quotient of the dominant Verma module. The answers for $L\left(s_{1}\right), L\left(s_{2}\right), L\left(s_{3}\right)$ and $L\left(s_{4}\right)$ are also positive by Corollary 21, and for $L\left(s_{1} s_{2} s_{1} s_{3} s_{2} s_{1} s_{4} s_{3} s_{2} s_{1}\right)$ the answer is positive as this module is a Verma module. The involutions

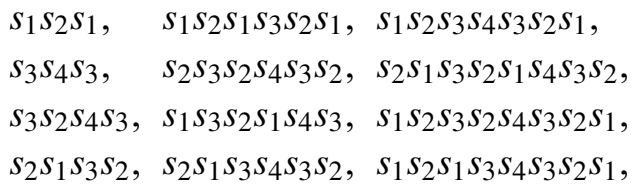


are all in left cells containing elements of the form $w_{0}^{\prime} w_{0}$ where $w_{0}^{\prime}$ is the longest element of some parabolic subgroup of $W$. Hence Kostant's problem has a positive answer for the corresponding simple modules by [Gabber and Joseph 1981a, Theorem 4.4] and [MS 2008a, Theorem 60]. The involutions $s_{2} s_{3} s_{4} s_{3} s_{2}$ and $s_{2} s_{4} s_{3} s_{2} s_{1}$ are both in left cells containing elements on the form $s w_{0}^{\prime} w_{0}$, where $w_{0}^{\prime}$ is the longest element of some parabolic subgroup, and $s$ is a simple reflection of the same parabolic subgroup, so Kostant's problem has a positive answer for $L\left(s_{2} s_{4} s_{3} s_{2} s_{1}\right)$ and $L\left(s_{2} s_{3} s_{4} s_{3} s_{2}\right)$ by [Mazorchuk 2005, Theorem 1] and [MS 2008a, Theorem 60]. Kostant's problem has a positive answer for $L\left(s_{1} s_{4}\right)$ and a negative answer for $L\left(s_{1} s_{3}\right)$ and $L\left(s_{2} s_{4}\right)$, by Corollary 22 .

Finally, the fact that Kostant's problem has a negative answer for $L\left(s_{2} s_{3} s_{2}\right)$, $L\left(s_{1} s_{3} s_{4} s_{3}\right)$ and $L\left(s_{1} s_{2} s_{1} s_{4}\right)$ follows from Theorem 14 by a direct computation as described in Remark 16. Consider first the involution $s_{2} s_{3} s_{2}$ for which we have $\mathfrak{a}\left(s_{2} s_{3} s_{2}\right)=3$. A direct calculation shows that the graded component $\mathrm{P}^{\hat{\boldsymbol{R}}}\left(s_{2} s_{3} s_{2}\right)_{2}$ has the following form after forgetting the grading:

$L\left(s_{3} s_{2}\right) \oplus L\left(s_{3} s_{2} s_{4} s_{3}\right) \oplus L\left(s_{2} s_{1} s_{3} s_{2} s_{4} s_{3}\right) \oplus L\left(s_{3} s_{2} s_{1} s_{4} s_{3} s_{2}\right)$

$\oplus L\left(s_{2} s_{3} s_{2} s_{1}\right) \oplus L\left(s_{2} s_{3}\right) \oplus L\left(s_{2} s_{1} s_{3} s_{2}\right) \oplus L\left(s_{2} s_{3} s_{2} s_{4}\right)$.

Another calculation shows that the graded component $\Delta(e)_{2}$ has the following form after forgetting the grading:

$$
\begin{aligned}
L\left(s_{3} s_{4}\right) \oplus L\left(s_{2} s_{4}\right) \oplus L\left(s_{2} s_{1}\right) \oplus L\left(s_{3} s_{2}\right) \oplus L\left(s_{1} s_{3}\right) \oplus L\left(s_{1} s_{4}\right) \\
\oplus L\left(s_{4} s_{3}\right) \oplus L\left(s_{1} s_{2}\right) \oplus L\left(s_{2} s_{3}\right) \oplus L\left(s_{2} s_{1} s_{3} s_{2}\right) \oplus L\left(s_{3} s_{2} s_{4} s_{3}\right) .
\end{aligned}
$$

Hence the module $L\left(s_{3} s_{2} s_{1} s_{4} s_{3} s_{2}\right)$ occurs in $\mathrm{P}^{\hat{R}}\left(s_{2} s_{3} s_{2}\right)_{2}$ but not in $\Delta(e)_{2}$. Note that $s_{3} s_{2} s_{1} s_{4} s_{3} s_{2}<\mathrm{R} s_{2} s_{3} s_{2}$. By Theorem 14 and Remark 15 this implies that Kostant's problem has a negative answer for $L\left(s_{2} s_{3} s_{2}\right)$.

For the involution $s_{1} s_{2} s_{1} s_{4}$ we have $\mathfrak{a}\left(s_{1} s_{2} s_{1} s_{4}\right)=4$. A direct calculation shows that the module $L\left(s_{1} s_{4} s_{3} s_{2} s_{1}\right)$ occurs in $\mathrm{P}^{\hat{R}}\left(s_{1} s_{2} s_{1} s_{4}\right)_{3}$ but not in $\Delta(e)_{3}$. Again, Remark 15 implies that Kostant's problem has a negative answer for $L\left(s_{1} s_{2} s_{1} s_{4}\right)$. Applying the symmetry of the root system we obtain that the answer for $L\left(s_{4} s_{3} s_{4} s_{1}\right)$ is also negative and it remains to observe that $s_{4} s_{3} s_{4} s_{1}=s_{1} s_{3} s_{4} s_{3}$.

The Robinson-Schensted correspondence associates to each $w \in S_{5}$ a pair

$$
(\alpha(x), \beta(x))
$$

of standard Young tableaux of the same shape [Sagan 2001, 3.1]. The shape defines a two-sided cell. Fixing $\alpha(x)$ or $\beta(x)$ defines a right or a left cell inside the twosided cell, respectively. We show on the next page the three two-sided cells of $S_{5}$ that contain left cells for elements of which Kostant's problem has a negative answer. The rows and columns in these figures are indexed by the corresponding $\alpha(x)$ 


\begin{tabular}{|c|c|c|c|c|c|}
\hline & \begin{tabular}{|l|l|l|}
1 & 2 & 3 \\
4 & 5 & \\
\cline { 1 - 2 } &
\end{tabular} & 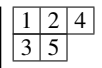 & \begin{tabular}{|l|l|l|}
1 & 3 & 4 \\
2 & 5 & \\
\end{tabular} & 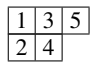 & \begin{tabular}{|l|l|l|}
1 & 2 & 5 \\
3 & 4 & \multicolumn{1}{|c}{} \\
\end{tabular} \\
\hline \begin{tabular}{|l|l|l|}
1 & 2 & 3 \\
4 & 5 & \\
\end{tabular} & 3243 & 324 & 3214 & 32143 & 321432 \\
\hline \begin{tabular}{|l|l|l|}
1 & 2 & 4 \\
3 & 5 & \multicolumn{1}{|c}{} \\
\end{tabular} & 243 & 24 & 214 & 2143 & 21432 \\
\hline 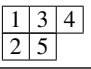 & 1321 & 124 & 14 & 143 & 1432 \\
\hline \begin{tabular}{|l|l|l|}
1 & 3 & 5 \\
2 & 4 & \\
\end{tabular} & 13243 & 1324 & 134 & 13 & 132 \\
\hline \begin{tabular}{|l|l|l|}
1 & 2 & 5 \\
3 & 4 & \multicolumn{1}{|c}{} \\
\end{tabular} & 213243 & 21324 & 2134 & 213 & 2132 \\
\hline
\end{tabular}

\begin{tabular}{|c|c|c|c|c|c|c|}
\hline & \begin{tabular}{|l|l|l|}
1 & 2 & 3 \\
4 & \\
5 & & \\
\end{tabular} & \begin{tabular}{|l|l|l|}
1 & 2 & 4 \\
\cline { 1 - 1 } & \multicolumn{1}{|l}{} \\
\cline { 1 - 1 } & \multicolumn{1}{|l}{} \\
\cline { 1 - 2 }
\end{tabular} & 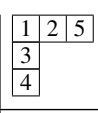 & \begin{tabular}{|l|l|l|}
1 & 3 & 4 \\
2 & \multicolumn{1}{|l}{} \\
\cline { 1 - 1 } 5 & \multicolumn{1}{|c|}{} \\
\cline { 1 - 2 }
\end{tabular} & \begin{tabular}{|l|l|l|}
1 & 3 & 5 \\
\cline { 1 - 1 } 2 & \multicolumn{1}{|l}{} \\
\cline { 1 - 1 } & \multicolumn{2}{|l}{} \\
\cline { 1 - 1 }
\end{tabular} & \begin{tabular}{|l|l|l|}
1 & 4 & 5 \\
\cline { 1 - 1 } 2 & \multicolumn{1}{|l}{} \\
\cline { 1 - 1 } & \multicolumn{2}{|l}{} \\
\cline { 1 - 2 }
\end{tabular} \\
\hline \begin{tabular}{|l|l|l|}
1 & 2 & 3 \\
4 & & \\
5 & \\
\end{tabular} & 343 & 3432 & 32432 & 34321 & 324321 & 3214321 \\
\hline \begin{tabular}{|l|l|l|}
1 & 2 & 4 \\
3 & \\
5 & \\
\end{tabular} & 2343 & 23432 & 2432 & 234321 & 24321 & 214321 \\
\hline \begin{tabular}{|l|l|l}
1 & 2 & 5 \\
3 & & \\
4 & \\
\end{tabular} & 23243 & 2324 & 232 & 23214 & 2321 & 21321 \\
\hline 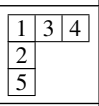 & 12343 & 123432 & 12432 & 1234321 & 124321 & 14321 \\
\hline 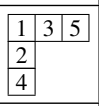 & 123243 & 12324 & 1232 & 123214 & 12321 & 1321 \\
\hline \begin{tabular}{|l|l|l}
1 & 4 & 5 \\
2 & \\
3 & \\
\end{tabular} & 1213243 & 121324 & 12132 & 12134 & 1213 & 121 \\
\hline
\end{tabular}

\begin{tabular}{|c|c|c|c|c|c|}
\hline & \begin{tabular}{|l|l|}
1 & 2 \\
3 & 4 \\
5 & \\
\end{tabular} & \begin{tabular}{|l|l|}
1 & 2 \\
3 & 5 \\
4 & \\
\end{tabular} & \begin{tabular}{|l|l|}
1 & 3 \\
2 & 4 \\
5 & \\
\end{tabular} & \begin{tabular}{|l|l|}
1 & 3 \\
2 & 5 \\
4 & \\
\end{tabular} & \begin{tabular}{|l|l|}
1 & 4 \\
2 & 5 \\
3 & \\
\end{tabular} \\
\hline \begin{tabular}{|l|l|}
\begin{tabular}{l|l}
$\mid l$ & 2 \\
5 & 4 \\
\end{tabular} \\
\end{tabular} & 213432 & 2132432 & 21343 & 21324321 & 2134321 \\
\hline \begin{tabular}{|l|l|}
1 & 2 \\
3 & 5 \\
4 & \\
\end{tabular} & 2321432 & 21321432 & 232143 & 2132143 & 213214 \\
\hline \begin{tabular}{|l|l|}
1 & 3 \\
2 & 4 \\
5
\end{tabular} & 13432 & 132432 & 1343 & 1324321 & 134321 \\
\hline \begin{tabular}{|l|l|}
1 & 3 \\
2 & 5 \\
3 & \\
\end{tabular} & 12321432 & 1321432 & 1232143 & 132143 & 13214 \\
\hline \begin{tabular}{|l|l|}
1 & 4 \\
2 & 5 \\
3 & \\
\end{tabular} & 1213432 & 121432 & 121343 & 12143 & 1214 \\
\hline
\end{tabular}


and $\beta(x)$, respectively. The left cells for which Kostant's problem has a negative answer are marked by arrows. Each element is denoted simply by the sequence of indices in some reduced expression, that is, $s_{1} s_{3} s_{2}$ is denoted by 132 . There seems to exist some hidden symmetry in these pictures, but we do not understand it yet.

4.7. Kostant's problem for $\mathfrak{s l}_{6}$. We are not able yet to give a complete answer to Kostant's problem in the case $\mathfrak{g}=\mathfrak{s l}_{6}$. The group $S_{6}$ has 76 involutions. For 45 involutions one can use arguments analogous to the arguments above to show that Kostant's problem has a positive answer; for 20 involutions one can analogously show that Kostant's problem has a negative answer. This leaves 11 involutions for which the answer is still unclear.

There are 39 involutions that lie in left cells containing an element of the form $w_{0}^{\prime} w_{0}$ or $s w_{0}^{\prime} w_{0}$, and hence Kostant's problem has a positive answer for these involutions. From Corollary 22 it follows that Kostant's problem has a positive answer for $L\left(s_{1} s_{4}\right), L\left(s_{1} s_{5}\right)$ and $L\left(s_{2} s_{5}\right)$.

The module $\theta_{w} L(\boldsymbol{w})$ is a quotient of an indecomposable projective module from 0 . The latter module has a Verma filtration. Classical results on inclusions of Verma modules [Dixmier 1996, Chapter 7] may be used in some cases to analyze the socle of the cokernel of $D^{\hat{R}} \hookrightarrow \theta_{w} L(\boldsymbol{w})$. Combined with Theorem 14, these arguments imply that Kostant's problem has a positive answer for $L\left(s_{2} s_{1} s_{3} s_{2}\right), L\left(s_{3} s_{2} s_{4} s_{3}\right)$ and $L\left(s_{4} s_{3} s_{5} s_{4}\right)$. We omit the details.

By Corollary 22, Kostant's problem has a negative answer for $L\left(s_{1} s_{2}\right), L\left(s_{2} s_{4}\right)$ and $L\left(s_{3} s_{5}\right)$. This, and computations as described in Remark 16, show that Kostant's problem has a negative answer for the following 17 involutions:

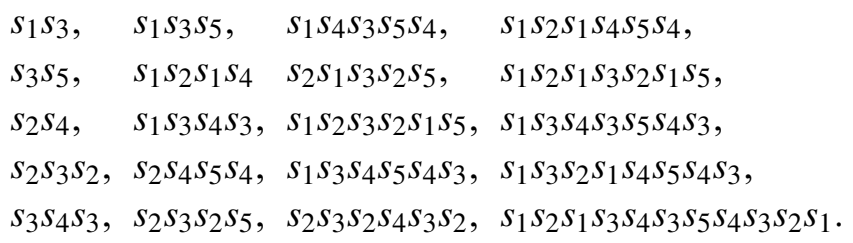

The remaining 11 involutions, which are not covered by Theorem 14, are:

$\begin{array}{lll}s_{1} s_{2} s_{1} s_{5}, & s_{2} s_{4} s_{3} s_{2} s_{5} s_{4}, & s_{1} s_{3} s_{2} s_{4} s_{3} s_{2} s_{1} s_{5} s_{4} s_{3}, \\ s_{1} s_{4} s_{5} s_{4}, & s_{2} s_{1} s_{4} s_{3} s_{2} s_{5} s_{4}, & s_{2} s_{1} s_{3} s_{2} s_{1} s_{4} s_{5} s_{4} s_{3} s_{2}, \\ s_{2} s_{3} s_{4} s_{3} s_{2}, & s_{1} s_{2} s_{3} s_{2} s_{4} s_{3} s_{2} s_{1}, & s_{2} s_{1} s_{3} s_{2} s_{4} s_{3} s_{2} s_{1} s_{5} s_{4} s_{3} s_{2} . \\ s_{2} s_{1} s_{3} s_{4} s_{3} s_{2}, & s_{2} s_{3} s_{2} s_{4} s_{5} s_{4} s_{3} s_{2} .\end{array}$

For these involutions the answer is still unclear. Some further progress in this case was recently made in [Kåhrström 2010]. 


\section{Acknowledgments}

Mazorchuk's research was partially supported by the Swedish Research Council. The authors thank the referee for very useful comments.

\section{References}

[Bernstein and Gel'fand 1980] J. N. Bernstein and S. I. Gel'fand, "Tensor products of finite- and infinite-dimensional representations of semisimple Lie algebras", Compositio Math. 41:2 (1980), 245-285. MR 82c:17003

[Bernstein et al. 1976] I. N. Bernstein, I. M. Gel'fand, and S. I. Gel'fand, “A certain category of $\mathfrak{g}$-modules”, Funkcional. Anal. i Priložen. 10:2 (1976), 1-8. In Russian; translated in Funct. Anal. Appl. 10 (1976), 87-92. MR 53 \#10880

[Björner and Brenti 2005] A. Björner and F. Brenti, Combinatorics of Coxeter groups, Grad. Texts in Math. 231, Springer, New York, 2005. MR 2006d:05001 Zbl 1110.05001

[Borho and Brylinski 1982] W. Borho and J.-L. Brylinski, "Differential operators on homogeneous spaces, I: Irreducibility of the associated variety for annihilators of induced modules", Invent. Math. 69:3 (1982), 437-476. MR 84b:17007

[Cline et al. 1988] E. Cline, B. Parshall, and L. Scott, "Finite dimensional algebras and highest weight categories", J. Reine Angew. Math. 391 (1988), 85-99. MR 90d:18005 Zbl 0657.18005

[Conze-Berline and Duflo 1977] N. Conze-Berline and M. Duflo, "Sur les représentations induites des groupes semi-simples complexes", Compositio Math. 34:3 (1977), 307-336. MR 55 \#12872 Zbl 0389.22016

[Dixmier 1996] J. Dixmier, Enveloping algebras, Grad. Studies in Math. 11, American Mathematical Society, Providence, RI, 1996. MR 97c:17010 Zbl 0867.17001

[Gabber and Joseph 1981a] O. Gabber and A. Joseph, "On the Bernšteĭn-Gel'fand-Gel'fand resolution and the Duflo sum formula", Compositio Math. 43:1 (1981), 107-131. MR 82k:17009

[Gabber and Joseph 1981b] O. Gabber and A. Joseph, "Towards the Kazhdan-Lusztig conjecture", Ann. Sci. École Norm. Sup. (4) 14:3 (1981), 261-302. MR 83e:17009 Zbl 0476.17005

[Irving 1985] R. S. Irving, "Projective modules in the category $0_{S}$ : self-duality", Trans. Amer. Math. Soc. 291:2 (1985), 701-732. MR 87i:17005 Zbl 0594.17005

[Jantzen 1983] J. C. Jantzen, Einhüllende Algebren halbeinfacher Lie-Algebren, Ergebnisse der Mathematik 3, Springer, Berlin, 1983. MR 86c:17011

[Joseph 1979] A. Joseph, " $W$-module structure in the primitive spectrum of the enveloping algebra of a semisimple Lie algebra", pp. 116-135 in Noncommutative harmonic analysis (Marseille, 1978), edited by J. Carmona and M. Vergne, Lecture Notes in Math. 728, Springer, Berlin, 1979. MR 80k:17007 Zbl 0422.17004

[Joseph 1980] A. Joseph, "Kostant's problem, Goldie rank and the Gel'fand-Kirillov conjecture", Invent. Math. 56:3 (1980), 191-213. MR 82f:17008

[Kåhrström 2010] J. Kåhrström, "Kostant's problem and parabolic subgroups”, Glasgow Math. J. 52:1 (2010), 19-32. Zbl 05656554

[Kazhdan and Lusztig 1979] D. Kazhdan and G. Lusztig, "Representations of Coxeter groups and Hecke algebras”, Invent. Math. 53:2 (1979), 165-184. MR 81j:20066 Zbl 0499.20035

[Khomenko and Mazorchuk 2004] O. Khomenko and V. Mazorchuk, "Structure of modules induced from simple modules with minimal annihilator", Canad. J. Math. 56:2 (2004), 293-309. MR 2005b:17012 Zbl 1071.17004 
[Khomenko and Mazorchuk 2005] O. Khomenko and V. Mazorchuk, "On Arkhipov's and Enright's functors", Math. Z. 249:2 (2005), 357-386. MR 2005k:17004 Zbl 1103.17002

[Lusztig 1985] G. Lusztig, "Cells in affine Weyl groups", pp. 255-287 in Algebraic groups and related topics (Kyoto/Nagoya, 1983), edited by R. Hotta, Adv. Stud. Pure Math. 6, North-Holland, Amsterdam, 1985. MR 87h:20074 Zbl 0569.20032

[Marietti 2006] M. Marietti, "Boolean elements in Kazhdan-Lusztig theory", J. Algebra 295:1 (2006), 1-26. MR 2006i:20004 Zbl 1097.20035

[Mazorchuk 2005] V. Mazorchuk, “A twisted approach to Kostant's problem”, Glasg. Math. J. 47:3 (2005), 549-561. MR 2007a:17018 Zbl 1081.17007

[Mazorchuk 2007] V. Mazorchuk, "Some homological properties of the category O", Pacific J. Math. 232:2 (2007), 313-341. MR 2008m:17013 Zbl 05366268

[Mazorchuk 2009] V. Mazorchuk, "Some homological properties of the category O, II", preprint, 2009. To appear in Rep. Theory. arXiv 0909.2729

[MS 2008a] V. Mazorchuk and C. Stroppel, "Categorification of (induced) cell modules and the rough structure of generalised Verma modules”, Adv. Math. 219:4 (2008), 1363-1426. MR 2010a: 20014 Zbl 05355944

[MS 2008b] V. Mazorchuk and C. Stroppel, "Categorification of Wedderburn's basis for $\mathbb{C}\left[S_{n}\right]$ ", Arch. Math. (Basel) 91:1 (2008), 1-11. MR 2009g:17006 Zbl 05323275

[MS 2008c] V. Mazorchuk and C. Stroppel, "Projective-injective modules, Serre functors and symmetric algebras", J. Reine Angew. Math. 616 (2008), 131-165. MR 2009e:16027 Zbl 05344019

[McGovern 1994] W. M. McGovern, "A remark on differential operator algebras and an equivalence of categories”, Compositio Math. 90:3 (1994), 305-313. MR 95a:17013 Zbl 0842.17009

[Miličić and Soergel 1997] D. Miličić and W. Soergel, "The composition series of modules induced from Whittaker modules", Comment. Math. Helv. 72:4 (1997), 503-520. MR 99e:17010 Zbl 0956.17004

[Ringel 1991] C. M. Ringel, "The category of modules with good filtrations over a quasi-hereditary algebra has almost split sequences”, Math. Z. 208:2 (1991), 209-223. MR 93c:16010 Zbl 0725. 16011

[Rocha-Caridi 1980] A. Rocha-Caridi, "Splitting criteria for $\mathfrak{g}$-modules induced from a parabolic and the Berňsteřn-Gel'fand-Gel'fand resolution of a finite-dimensional, irreducible $\mathfrak{g}$-module", Trans. Amer. Math. Soc. 262:2 (1980), 335-366. MR 82f:17006 Zbl 0449.17008

[Sagan 2001] B. E. Sagan, The symmetric group: representations, combinatorial algorithms, and symmetric functions, 2nd ed., Grad. Texts in Math. 203, Springer, New York, 2001. MR 2001m: 05261 Zbl 0964.05070

[Soergel 1990] W. Soergel, "Kategorie O, perverse Garben und Moduln über den Koinvarianten zur Weylgruppe”, J. Amer. Math. Soc. 3:2 (1990), 421-445. MR 91e:17007

[Stroppel 2003] C. Stroppel, "Category O: gradings and translation functors", J. Algebra 268:1 (2003), 301-326. MR 2004i:17007 Zbl 1040.17002

[Vogan 1979] D. A. Vogan, Jr., "Irreducible characters of semisimple Lie groups, II: The KazhdanLusztig conjectures", Duke Math. J. 46:4 (1979), 805-859. MR 81f:22024

Communicated by Georgia Benkart

Received 2008-06-18 Revised 2009-10-17 Accepted 2009-12-31

Johan.Karstrom@gmail.com Department of Mathematics, Uppsala University, 75106 Uppsala, Sweden 
mazor@math.uu.se

Department of Mathematics, Uppsala University, 75106 Uppsala, Sweden

http://www.math.uu.se/ mazor/ 